\title{
Detection of Ideal Resource for Multiqubit Teleportation
}

\author{
Ming-Jing Zhao ${ }^{1}$, Bin $\mathrm{Chen}^{2}$, and Shao-Ming $\mathrm{Fei}^{2,3}$ \\ ${ }^{1}$ Department of Mathematics, School of Science, \\ Beijing Information Science and Technology University, \\ 100192, Beijing, China \\ ${ }^{2}$ School of Mathematical Sciences, \\ Capital Normal University, \\ Beijing 100048, China \\ 3 Max-Planck-Institute for Mathematics in the Sciences, \\ 04103 Leipzig, Germany
}

\begin{abstract}
We give a sufficient condition in detecting entanglement resource for perfect multiqubit teleportation. The criterion involves only local measurements on some complementary observables and can be experimentally implemented. It is also a necessary condition for fully separability of multiqubit states. Moreover, by proving the optimality of teleportation witnesses, we solve the open problem in [Phys. Rev. A 86, 032315 (2012)].
\end{abstract}

PACS numbers: 03.65.Ud, 03.67.Mn 


\section{INTRODUCTION}

Quantum teleportation, employing classical communication and shared resource of entanglement, allows to transmit an unknown quantum state from a sender to a receiver that are spatially separated. The first teleportation protocol was proposed by using EinsteinPodolsky-Rosen pair by Bennett et. al. [1]. Then the three-qubit GHZ state and a class of W states are revealed to be the ideal resource for faithful teleportation of one-qubit state [2, 3]. For two-qubit state teleportation, the tensor product of two Bell states [4] and the genuine four-qubit entangled state [5] are showed to have the ability of faithful teleportation. For three-qubit state, Refs. [6, 7] have investigated its teleportation in terms of a genuine entangled six-qubit state. Some entangled $2 n$-qubit states have been presented for $n$-qubit state teleportation [8-10] .

One important problem related to quantum teleportation is how to know if a general quantum state is the ideal resource for faithful teleportation, or is useful for teleportation with fidelity better than classical states. For bipartite case, it has been shown that the quantum states which are not useful for quantum teleportation compose a compact convex set and a teleportation witness has been presented for the first time in Ref. [11]. Then a complete set of teleportation witness to detect all the ideal resource for teleportation is constructed in Ref. [12]. In Ref. [13], a systematic method to construct teleportation witness from entanglement witness is provided. The properties of teleportation witness are further studied in Ref. [14]. For multipartite case, Ref. [15] provides the necessary and sufficient condition that the genuine $2 n$-qubit entanglement channels must satisfy for teleporting an arbitrary $n$-qubit state, and Refs. [16, 17] analyze the criterion of multiqubit states for $n$-qubit teleportation.

In this paper, we first study the multiqubit teleportation and propose a linear operator to detect ideal resource for multiqubit teleportation. This operator is expressed by the local complementary observables. It gives a sufficient condition for the detection of all ideal resource for multiqubit teleportation. This operator can also be used to detect multiqubit entanglement and serves as a necessary condition for fully separability. Then we analyze teleportation witness for bipartite high dimensional case. Moreover, the problem left in Ref. [13] is solved by explaining the optimality of teleportation witness. 


\section{DETECTION OF IDEAL RESOURCE FOR MULTIQUBIT TELEPORTATION}

We first consider $n$-qubit systems. Let $\vec{a}_{1}, \vec{a}_{2}$ and $\vec{a}_{3}$ be three dimensional unit vectors. We say that three observables $X_{k}=\vec{a}_{k} \cdot \vec{\sigma}, k=1,2,3$, are complementary if they satisfy $X_{1} X_{2} X_{3}=-\mathrm{i} I_{2}$, where $\mathrm{i}=\sqrt{-1}, I_{2}$ is the $2 \times 2$ identity matrix, $\vec{\sigma}=\left(\sigma_{1}, \sigma_{2}, \sigma_{3}\right), \sigma_{1}=|0\rangle\langle 1|+$ $|1\rangle\left\langle 0\left|, \sigma_{2}=\mathrm{i}(|0\rangle\langle 1|-| 1\rangle\langle 0|), \sigma_{3}=\right| 0\right\rangle\langle 0|-| 1\rangle\langle 1|$ are Pauli matrices, which means that the orientation of the basis formed by three real vectors $\left\{\vec{a}_{k}\right\}_{k=1}^{3}$ is right-handed, with the same orientation as that of three Pauli matrices. Let $\left\{X_{k}^{A_{i}}\right\}_{k=1}^{3}, i=1, \cdots, n$, be complementary observables acting on $A_{i}$. We define the operator,

$$
\begin{aligned}
& \Gamma \equiv \frac{1}{2^{2 n}}\left\{1+\sum_{i}\left[X_{1}^{A_{i}} \otimes \sigma_{1}^{B_{i}}-X_{2}^{A_{i}} \otimes \sigma_{2}^{B_{i}}+X_{3}^{A_{i}} \otimes \sigma_{3}^{B_{i}}\right]\right. \\
& +\sum_{i_{1}, i_{2}}\left[\sum_{j_{1}, j_{2}=1,3} X_{j_{1}}^{A_{i_{1}}} \otimes X_{j_{2}}^{A_{i_{2}}} \otimes \sigma_{j_{1}}^{B_{i_{1}}} \otimes \sigma_{j_{2}}^{B_{i_{2}}}-\sum_{j=1,3} X_{j}^{A_{i_{1}}} \otimes X_{2}^{A_{i_{2}}} \otimes \sigma_{j}^{B_{i_{1}}} \otimes \sigma_{2}^{B_{i_{2}}}\right. \\
& \left.+X_{2}^{A_{i_{1}}} \otimes X_{2}^{A_{i_{2}}} \otimes \sigma_{2}^{B_{i_{1}}} \otimes \sigma_{2}^{B_{i_{2}}}\right] \\
& +\cdots+\sum_{i_{1}, \cdots, i_{s}}\left[\sum_{j_{1}, \cdots, j_{s}=1,3} X_{j_{1}}^{A_{i_{1}}} \otimes \cdots \otimes X_{j_{s}}^{A_{i_{s}}} \otimes \sigma_{j_{1}}^{B_{i_{1}}} \otimes \cdots \otimes \sigma_{j_{s}}^{B_{i_{s}}}\right. \\
& -\sum_{j_{1}, \cdots, j_{s-1}=1,3} X_{j_{1}}^{A_{i_{1}}} \otimes \cdots \otimes X_{j_{s-1}}^{A_{i_{s-1}}} \otimes X_{2}^{A_{i_{s}}} \otimes \sigma_{j_{1}}^{B_{i_{1}}} \otimes \cdots \otimes \sigma_{j_{s-1}}^{B_{i_{s-1}}} \otimes \sigma_{2}^{B_{i_{s}}}+\cdots \\
& \left.+(-1)^{s} X_{2}^{A_{i_{1}}} \otimes \cdots \otimes X_{2}^{A_{i_{s}}} \otimes \sigma_{2}^{B_{i_{1}}} \otimes \cdots \otimes \sigma_{2}^{B_{i_{s}}}\right] \\
& +\cdots+\left[\sum_{j_{1}, \cdots, j_{n}=1,3} X_{j_{1}}^{A_{1}} \otimes \cdots \otimes X_{j_{n}}^{A_{n}} \otimes \sigma_{j_{1}}^{B_{1}} \otimes \cdots \otimes \sigma_{j_{n}}^{B_{n}}\right. \\
& -\sum_{i_{1}, \cdots, i_{n}} \sum_{j_{1}, \cdots, j_{n-1}=1,3}^{X_{j_{1}}} X_{i_{1}}^{A_{1}} \otimes \cdots \otimes X_{j_{n-1}}^{A_{i_{n-1}}} \otimes X_{2}^{A_{i_{n}}} \otimes \sigma_{j_{1}}^{B_{i_{1}}} \otimes \cdots \otimes \sigma_{j_{n-1}}^{B_{i_{n-1}}} \otimes \sigma_{2}^{B_{i_{n}}} \\
& \left.\left.+\cdots+(-1)^{n} X_{2}^{A_{1}} \otimes \cdots \otimes X_{2}^{A_{n}} \otimes \sigma_{2}^{B_{1}} \otimes \cdots \otimes \sigma_{2}^{B_{n}}\right]\right\} .
\end{aligned}
$$

The operator $\Gamma$ can be used to detect the ideal resources for $n$-qubit teleportation.

Theorem 1. Any 2n-qubit pure state $\rho=|\phi\rangle_{A_{1} \cdots A_{n}, B_{1} \cdots B_{n}}\langle\phi|$, with qubits $A_{i}$ in $A$ part and qubits $B_{i}$ in $B$ part respectively, can be used to teleport an arbitrary $n$-qubit state faithfully if there exist complementary observables $\left\{X_{k}^{A_{i}}\right\}_{k=1}^{3}$ acting on $A_{i}$ such that $\langle\Gamma\rangle_{\rho}=1$.

[Proof]. First, as is well known, the tensor product of Bell states,

$$
\left|\phi^{+}\right\rangle_{A_{1} \cdots A_{n}, B_{1} \cdots B_{n}}=\frac{1}{\sqrt{2^{n}}} \sum_{i_{1}, \cdots, i_{n}=0,1}\left|i_{1}, \cdots, i_{n}\right\rangle_{A_{1} \cdots A_{n}}\left|i_{1}, \cdots, i_{n}\right\rangle_{B_{1} \cdots B_{n}}
$$

is an ideal resource for multiqubit teleportation. Let us expand the operator $\left|\phi^{+}\right\rangle_{A_{1} \cdots A_{n}, B_{1} \cdots B_{n}}\left\langle\phi^{+}\right|$ in terms of the Pauli operators. Note that the expectation values of $\sigma_{k}$ with respect to $\left|\phi^{+}\right\rangle_{A_{1} \cdots A_{n}, B_{1} \cdots B_{n}}\left\langle\phi^{+}\right|$are all zero expect for the case that there are even number of $\sigma_{k} \mathrm{~s}$, with 
one half acting on some qubits of $A$ part and the other half acting on the corresponding qubits of $B$ part, namely,

$$
\operatorname{Tr}\left(\sigma_{k}^{A_{i_{1}}} \otimes \cdots \otimes \sigma_{k}^{A_{i_{s}}} \otimes \sigma_{k}^{B_{i_{1}}} \otimes \cdots \otimes \sigma_{k}^{B_{i_{s}}}\left|\phi^{+}\right\rangle_{A_{1} \cdots A_{n}, B_{1} \cdots B_{n}}\left\langle\phi^{+}\right|\right)=1,
$$

$k=1,3$, and

$$
\operatorname{Tr}\left(\sigma_{2}^{A_{i_{1}}} \otimes \cdots \otimes \sigma_{2}^{A_{i_{s}}} \otimes \sigma_{2}^{B_{i_{1}}} \otimes \cdots \otimes \sigma_{2}^{B_{i_{s}}}\left|\phi^{+}\right\rangle_{A_{1} \cdots A_{n}, B_{1} \cdots B_{n}}\left\langle\phi^{+}\right|\right)=(-1)^{s},
$$

$s=1,2, \cdots, n$. For the case of different Pauli operators acting on the same $t$-th qubit in $A$ and $B$ parts, consider $\sigma_{k}$ acts on the qubit $A_{t}$ and $\sigma_{k^{\prime}}$ acts on the qubit $B_{t}$. Then the expectation value

$$
\operatorname{Tr}\left(\sigma_{i_{1}}^{A_{1}} \otimes \cdots \otimes \sigma_{k}^{A_{t}} \otimes \cdots \otimes \sigma_{i_{n}}^{A_{n}} \otimes \sigma_{j_{1}}^{B_{1}} \otimes \cdots \otimes \sigma_{k^{\prime}}^{B_{t}} \otimes \cdots \otimes \sigma_{j_{n}}^{B_{n}}\left|\phi^{+}\right\rangle_{A_{1} \cdots A_{n}, B_{1} \cdots B_{n}}\left\langle\phi^{+}\right|\right)=0
$$

due to $\sum_{i_{t}, j_{t}=0,1}\left\langle i_{t}\left|\sigma_{k}\right| j_{t}\right\rangle\left\langle i_{t}\left|\sigma_{k^{\prime}}\right| j_{t}\right\rangle=0$ for $k \neq k^{\prime}, k, k^{\prime}=0,1,2,3$, and $\sigma_{0}$ is the identity operator. From the above analysis, we have

$$
\begin{aligned}
& \left|\phi^{+}\right\rangle_{A_{1} \cdots A_{n}, B_{1} \cdots B_{n}}\left\langle\phi^{+}\right|=\frac{1}{2^{2 n}}\left\{1+\sum_{i}\left[\sigma_{1}^{A_{i}} \otimes \sigma_{1}^{B_{i}}-\sigma_{2}^{A_{i}} \otimes \sigma_{2}^{B_{i}}+\sigma_{3}^{A_{i}} \otimes \sigma_{3}^{B_{i}}\right]\right. \\
& +\sum_{i_{1}, i_{2}}\left[\sum_{j_{1}, j_{2}=1,3} \sigma_{j_{1}}^{A_{i_{1}}} \otimes \sigma_{j_{2}}^{A_{i_{2}}} \otimes \sigma_{j_{1}}^{B_{i_{1}}} \otimes \sigma_{j_{2}}^{B_{i_{2}}}-\sum_{j=1,3} \sigma_{j}^{A_{i_{1}}} \otimes \sigma_{2}^{A_{i_{2}}} \otimes \sigma_{j}^{B_{i_{1}}} \otimes \sigma_{2}^{B_{i_{2}}}\right. \\
& \left.+\sigma_{2}^{A_{i_{1}}} \otimes \sigma_{2}^{A_{i_{2}}} \otimes \sigma_{2}^{B_{i_{1}}} \otimes \sigma_{2}^{B_{i_{2}}}\right] \\
& +\cdots+\sum_{i_{1}, \cdots, i_{s}}\left[\sum_{j_{1}, \cdots, j_{s}=1,3} \sigma_{j_{1}}^{A_{i_{1}}} \otimes \cdots \otimes \sigma_{j_{s}}^{A_{i_{s}}} \otimes \sigma_{j_{1}}^{B_{i_{1}}} \otimes \cdots \otimes \sigma_{j_{s}}^{B_{i_{s}}}\right. \\
& \quad \sum_{j_{1}, \cdots, j_{s-1}=1,3} \sigma_{j_{1}}^{A_{i_{1}}} \otimes \cdots \otimes \sigma_{j_{s-1}}^{A_{i_{s}-1}} \otimes \sigma_{2}^{A_{i_{s}}} \otimes \sigma_{j_{1}}^{B_{i_{1}}} \otimes \cdots \otimes \sigma_{j_{s-1}}^{B_{i_{s}-1}} \otimes \sigma_{2}^{B_{i_{s}}}+\cdots \\
& \left.+(-1)^{s} \sigma_{2}^{A_{i_{1}}} \otimes \cdots \otimes \sigma_{2}^{A_{i_{s}}} \otimes \sigma_{2}^{B_{i_{1}}} \otimes \cdots \otimes \sigma_{2}^{B_{i_{s}}}\right] \\
& +\cdots+\left[\sum_{j_{1}, \cdots, j_{n}=1,3} \sigma_{j_{1}}^{A_{1}} \otimes \cdots \otimes \sigma_{j_{n}}^{A_{n}} \otimes \sigma_{j_{1}}^{B_{1}} \otimes \cdots \otimes \sigma_{j_{n}}^{B_{n}}\right. \\
& -\sum_{i_{1}, \cdots, i_{n}} \sum_{j_{1}, \cdots, j_{n-1}=1,3} \sigma_{j_{1}}^{A_{i_{1}}} \otimes \cdots \otimes \sigma_{j_{n-1}}^{A_{i_{n-1}}} \otimes \sigma_{2}^{A_{i_{n}}} \otimes \sigma_{j_{1}}^{B_{i_{1}}} \otimes \cdots \otimes \sigma_{j_{n-1}}^{B_{i_{n-1}}} \otimes \sigma_{2}^{B_{i_{n}}} \\
& \left.\left.+\cdots+(-1)^{n} \sigma_{2}^{A_{1}} \otimes \cdots \otimes \sigma_{2}^{A_{n}} \otimes \sigma_{2}^{B_{1}} \otimes \cdots \otimes \sigma_{2}^{B_{n}}\right]\right\} .
\end{aligned}
$$

Denote $\left(U^{A_{i}}\right)^{\dagger} \sigma_{k} U^{A_{i}}=X_{k}^{A_{i}}$. Then $\left\{X_{k}^{A_{i}}\right\}_{k=1}^{3}$ are complementary observables satisfying $X_{1}^{A_{i}} X_{2}^{A_{i}} X_{3}^{A_{i}}=-\mathrm{i} I_{2}, i=1,2, \cdots, n$. The unitary operators and the complementary observables can be mutually determined uniquely. If $\langle\Gamma\rangle_{\rho}=1$ for some local complementary observables, then there exist unitary operators $U^{A_{1}}, \cdots, U^{A_{n}}$ such that

$$
U^{A_{1}} \otimes \cdots \otimes U^{A_{n}}|\phi\rangle_{A_{1} \cdots A_{n}, B_{1} \cdots B_{n}}\langle\phi| U^{A_{1} \dagger} \otimes \cdots \otimes U^{A_{n} \dagger}
$$


is of the form (2). Therefore, $\rho$ is local unitarily equivalent to $\left|\phi^{+}\right\rangle_{A_{1} \cdots A_{n}, B_{1} \cdots B_{n}}\left\langle\phi^{+}\right|$and is an ideal resource for multiqubit teleportation.

Theorem 1 provides a method to detect ideal resource for multiqubit teleportation. All quantum states $|\phi\rangle_{A_{1} \cdots A_{n}, B_{1} \cdots B_{n}}$ that can be detected by Theorem 1 are local unitarily equivalent to $\left|\phi^{+}\right\rangle_{A_{1} \cdots A_{n}, B_{1} \cdots B_{n}}$. And if a quantum state is local unitarily equivalent to $\left|\phi^{+}\right\rangle_{A_{1} \cdots A_{n}, B_{1} \cdots B_{n}}$, then it must satisfy $\langle\Gamma\rangle_{\rho}=1$ for some local complementary observables $\left\{X_{k}^{A_{i}}\right\}_{k=1}^{3}$, since $U^{A_{1}} \otimes \cdots \otimes U^{A_{n}} \otimes U^{B_{1}} \otimes \cdots \otimes U^{B_{n}}\left|\phi^{+}\right\rangle=U^{A_{1}}\left(U^{B_{1}}\right)^{T} \otimes \cdots \otimes U^{A_{n}}\left(U^{B_{n}}\right)^{T}\left|\phi^{+}\right\rangle$ in calculation of the mean values of observables. Although Theorem 1 only gives a sufficient condition for the detection of multiqubit teleportation resource, it is experimentally feasible. Moreover, one only needs to measure different local complementary observables on $A$ part and the observables on $B$ part are fixed. For example, to detect $\left|\phi^{+}\right\rangle_{A_{1} \cdots A_{n}, B_{1} \cdots B_{n}}$, one simply chooses $X_{k}=\sigma_{k}, k=1,2,3$. Theorem 1 may also help in the characterization of teleportation witness in multiqubit systems.

Corollary 1. If a $2 n$-qubit mixed state $\rho$ is fully separable, then $\langle\Gamma\rangle_{\rho} \leq \frac{1}{2^{n}}$ for all complementary observables $\left\{X_{k}^{A_{i}}\right\}_{k=1}^{3}$ acting on the qubit $A_{i}, i=1, \cdots, n$.

[Proof]. Due to the linearity of the operator $\Gamma$, we only need to prove $\langle\Gamma\rangle_{\rho} \leq \frac{1}{2^{n}}$ for all pure

fully separable states $|\psi\rangle$. By proving that the expectation value $\left\langle\mid \phi^{+}\right\rangle_{A_{1} \cdots A_{n}, B_{1} \cdots B_{n}}\left\langle\phi^{+} \mid\right\rangle_{|\psi\rangle\langle\psi|} \leq$ $\frac{1}{2^{n}}$, one can prove the corollary directly.

Corollary 1 gives a necessary condition for fully separable state. It shows that if the expectation value of $\Gamma$ is more than $\frac{1}{2^{n}}$ for some complementary observables, then quantum state is entangled.

\section{BIPARTITE TELEPORTATION WITNESS}

Now we consider the detection of ideal resource for teleportation of high dimensional systems. Let $H_{n}$ be an $n$-dimensional complex vector space, with $\{|i\rangle\}_{i=0}^{n-1}$ an orthonormal basis. Let $\rho$ be a density matrix in $H_{n} \otimes H_{n}$. The optimal fidelity of teleportation with $\rho$ as the entangled resource is given by [18-20]

$$
f_{\max }(\rho)=\frac{n F(\rho)}{n+1}+\frac{1}{n+1} .
$$

$F(\rho)$ is the fully entangled fraction with respect to $\rho$ :

$$
F(\rho)=\max _{U}\left\langle\psi^{+}\left|\left(U^{\dagger} \otimes I_{n}\right) \rho\left(U \otimes I_{n}\right)\right| \psi^{+}\right\rangle,
$$


where $U$ is any $n \times n$ unitary matrix, $I_{n}$ is the $n \times n$ identity matrix, and $\left|\psi^{+}\right\rangle$is the maximally entangled state,

$$
\left|\psi^{+}\right\rangle=\frac{1}{\sqrt{n}} \sum_{i=0}^{n-1}|i i\rangle .
$$

A state $\rho$ is a useful resource for teleportation if and only if $F(\rho)>\frac{1}{n}[19]$. If $F(\rho) \leq \frac{1}{n}$, the fidelity (3) is no better than separable states. Recently in Ref. [11], the authors show that the set of states which are not useful for quantum teleportation, i.e. their fully entangled fractions are no more than $\frac{1}{n}$, is also convex and compact. Therefore there exist witness operators which detect some entangled states that are useful for teleportation.

A teleportation witness $W$ is a Hermitian operator such that (i) $\operatorname{Tr}(W \rho) \geq 0$ for all states $\rho$ that are not useful for quantum teleportation. (ii) there exists at least one entangled state $\rho$ which is useful for teleportation such that $\operatorname{Tr}(W \rho)<0$ [11, 13]. For entangled state $\rho$, if $\operatorname{Tr}(W \rho)<0$, then teleportation witness $W$ could detect $\rho$ as a useful resource for teleportation. Between two teleportation witnesses $W_{1}$ and $W_{2}, W_{1}$ is said to be finer than $W_{2}$ if $W_{1}$ could detect all the entangled states which could be detected by $W_{2}$. A witness is said to be optimal if there exist no other witnesses that are finer than it [21]. It is obvious that any teleportation witness is also an entanglement witness.

Since all separable states are positive under partial transpositions (PPT) and there exist PPT entangled states, therefore the set of all separable states is a proper subset of the set of PPT states. Similarly, since all PPT states are not useful in teleportation [19] and there exist nonpositive partial transpositions (NPT) entangled states that are also not useful in teleportation [22], the set of PPT states is again a proper subset of the set of states that are not useful in the teleportation. A teleportation witness is a hyperplane that separates a point which is an entangled state that is useful for quantum teleportation from the convex and compact set of states that are not useful in teleportation. An optimal teleportation witness is then a hyperplane that is tangent to the set of states that are not useful in the teleportation (see Fig. 1).

Now we consider the teleportation witness

$$
W(I)=\frac{1}{n} I-\left|\psi^{+}\right\rangle\left\langle\psi^{+}\right| .
$$




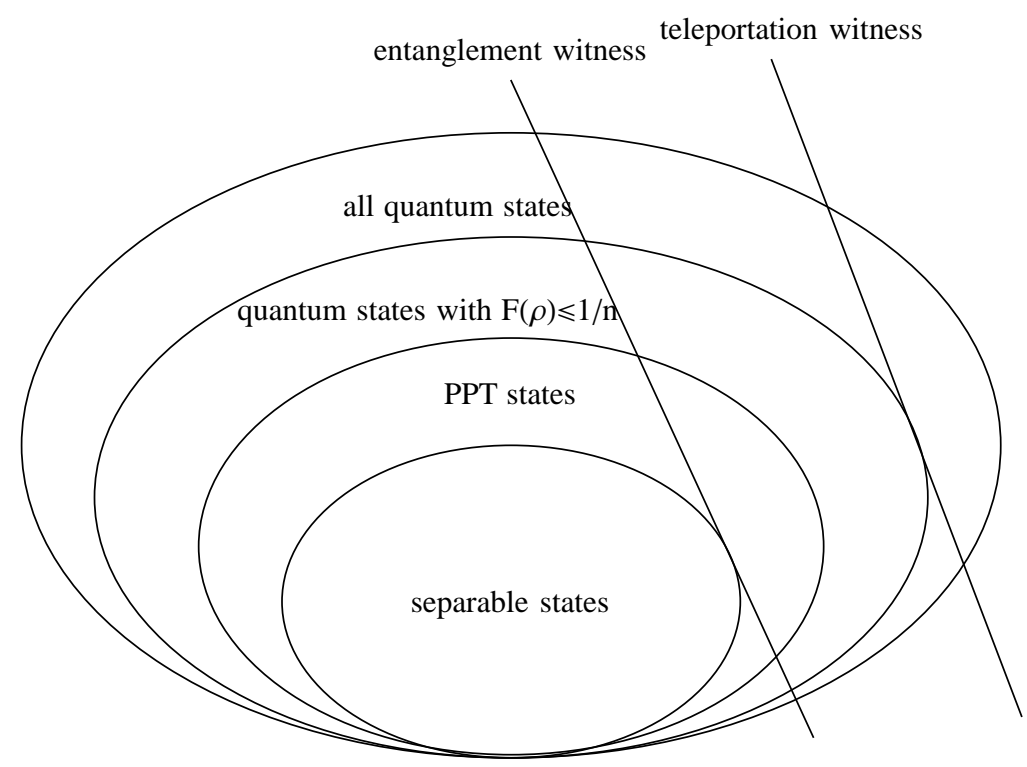

FIG. 1.

$W(I)$ is a teleportation witness in the sense that for arbitrary states $\rho$ which are not useful for quantum teleportation, i.e, $F(\rho) \leq \frac{1}{n}$, then $\operatorname{Tr}(W(I) \rho) \geq 0$. And $\operatorname{Tr}\left(W(I)\left|\psi^{+}\right\rangle\left\langle\psi^{+}\right|\right)<0$ for the ideal teleportation resource $\left|\psi^{+}\right\rangle\left\langle\psi^{+}\right|$. Furthermore $W(I)$ is an optimal teleportation witness. Consider the following product vectors:

$$
\begin{aligned}
K_{j} & =|j j\rangle, \\
K_{k l} & =(|k\rangle+|l\rangle) \otimes(|k\rangle+|l\rangle), \\
K_{k l}^{\prime} & =(|k\rangle+\mathrm{i}|l\rangle) \otimes(|k\rangle-\mathrm{i}|l\rangle),
\end{aligned}
$$

with $j, k, l=0,1, \cdots, n-1$ and $k<l$. These $d^{2}$ product vectors are linear independent and satisfy $\operatorname{Tr}\left(W(I) K_{j}\right)=0, \operatorname{Tr}\left(W(I) K_{k l}\right)=0, \operatorname{Tr}\left(W(I) K_{k l}^{\prime}\right)=0$. Note that if a set of product vectors $P_{W}=\{|e, f\rangle: \operatorname{Tr}(W|e, f\rangle\langle e, f|)=0\}$ spans the relevant product vector space, then $W$ is an optimal entanglement witness [21]. Since an optimal entanglement witness is surly an optimal teleportation witness, $W(I)$ must be an optimal teleportation witness. In Ref. [13], a teleportation witness for bipartite system is given and its optimality is proved for two dimensional case and that for high dimensional is left. In fact, one can calculate that $W(I)$ is the exact teleportation witness in Ref. [13]. In this way, the problem proposed in Ref. [13] is solved by proving the optimality of the teleportation witness $W(I)$.

In fact, teleportation witness $W(I)$ is an entanglement witness of the form $W=\alpha I-$ $|\psi\rangle\langle\psi|$ with $\alpha=\max _{|\phi\rangle=|a\rangle \otimes|b\rangle}|\langle\psi \mid \phi\rangle|^{2}$, where $|\psi\rangle$ is an entangled pure state. Such entangle- 
ment witness $W$ could detect the entanglement of $|\psi\rangle$, and the entanglement of NPT states. The distance between the entangled state $|\psi\rangle$ and the set of separable states is $\alpha$. Especially, the distance between the maximally entangled state $\left|\psi^{+}\right\rangle$and the set of separable states is $\frac{1}{n}$. The distance between $\left|\psi^{+}\right\rangle$and the set of PPT states is also $\frac{1}{n}$.

Now we define the teleportation witness

$$
W(U)=\frac{1}{n} I-U \otimes I\left|\psi^{+}\right\rangle\left\langle\psi^{+}\right| U^{\dagger} \otimes I
$$

One can verify that $W(U)$ is an optimal teleportation witness for arbitrary unitary operator $U$. Furthermore, the set of teleportation witnesses $\{W(U)\}$ is complete since for arbitrary entangled state $\rho$ that is useful for teleportation, there exists a unitary operator $U$ such that $\operatorname{Tr}(W(U) \rho)<0$.

\section{CONCLUSION}

We have presented an experimental approach to detect the ideal resource for quantum teleportation for multiqubit systems, by deriving a condition to detect the local unitary equivalence of the tensor product of Bell states. Our criterion only involves local measurements on complementary observables in half part of a system. It also helps in characterizing the teleportation witness for multiqubit systems [14]. For bipartite high dimensional systems, we have analyzed the teleportation witnesses. Moreover, we have solved the open problem presented in Ref. [13].

[1] Bennett C H, Brassard G, Crépeau C, Jozsa R, Peres A, and Wootters W K 1993 Phys. Rev. Lett. 70, 1895

[2] Karlsson A and Bourennane M 1998 Phys. Rev. A 58, 4394

[3] Agrawal P and Pati A 2006 Phys. Rev. A 74, 062320

[4] Rigolin G 2005 Phys. Rev. A 71, 032303

[5] Yeo Y and Chua W K 2006 Phys. Rev. Lett. 96, 060502

[6] Choudhury S, Muralidharan S, and Panigrahi P K 2009 J. Phys. A: Math. Theor. 42, 115303

[7] Yin X, Liu Y, Zhang Z, Zhang W, and Zhang Z 2010 Sci. China Ser G-Phys. Mech. Astron. 53, 2059 
[8] Muralidharan S, Karumanchi S, Jain S, Srikanth R, and Panigrahi P K 2011 Eur. Phys. J. D. 61, 757

[9] Zhao M J, Li Z G, Li-Jost X, and Fei S M 2012 J. Phys. A, 45, 405303

[10] Wang M Y and Yan F L 2011 Chin. Phys. B 20, 120309

[11] Ganguly N, Adhikari S, Majumdar A S, and Chatterjee J 2011 Phys. Rev. Lett. 107, 270501

[12] Zhao M J, Fei S M, and Li-Jost X 2012 Phys. Rev. A 85, 054301

[13] Adhikari S, Ganguly N, and Majumdar A S 2012 Phys. Rev. A 86, 032315

[14] Kumar A, Adhikari S, Agrawal P 2013 Quantum Inf. Process 12, 2475

[15] Chen P X, Zhu S Y, and Guo G C 2006 Phys. Rev. A 74, 032324

[16] Cheung C Y and Zhang Z J 2009 Phys. Rev. A 80, 022327

[17] Liu D M, Wang Y W, Jiang X M, and Zheng Y Z 2010 Chin. Phys. B 19, 020307

[18] Bennett C H, DiVincenzo D P, Smolin J A, and Wootters W K 1996 Phys. Rev. A 54, 3824

[19] Horodecki M, Horodecki P, and Horodecki R 1999 Phys. Rev. A 60, 1888

[20] Albeverio S, Fei S M, and Yang W L 2002 Phys. Rev. A 66, 012301

[21] Lewenstein M, Kraus B, Cirac J I, and Horodecki P 2000 Phys. Rev. A 62, 052310

[22] Badziąg P, Horodecki M, Horodecki P, and Horodecki R 2000 Phys. Rev. A 62, 012311 